J. Clin. Chem. Clin. Biochem.

Vol. 18, 1980, pp. 297-301

\title{
Tageszeitliche Änderungen erhöhter Aktivitäten der sauren Phosphatase beim Prostatacarcinom ${ }^{1}$ )
}

\author{
Von H. Wisser, E. Knoll
}

Abteilung für Klinische Chemie und

\section{G. Schmid}

\author{
Abteilung für Nephrologie und Rheumatologie \\ Robert-Bosch-Krankenhaus, Stuttgart
}

(Eingegangen am 24. Oktober/8. Dezember 1979)

Zusammenfassung: Bei Patienten mit Prostatacarcinom mit erhöhten Aktivitäten der gesamten sauren Phosphatase und der Prostataphosphatase wurde die Frage einer Tagesrhythmik dieser Enzymaktivitäten untersucht. Die Aktivitäten der gesamten sauren Phosphatase, deren Tartrat-hemmbaren Anteile und der Lactatdehydrogenase, die bei diesen Patienten erhöht sind, fallen nach Therapie ab, während die Aktivität der alkalischen Phosphatase ansteigt. Bei allen Patienten mit Prostatacarcinom zeigen die gesamte und die Tartrat-hemmbare saure Phosphatase sowie das Cortisol vor Therapie eine Tageszeitabhängigkeit mit einem Minimum in der Nachtzeit. Diese Tageszeitabhängigkeit ist bei einem Patienten nach Orchiek tomie - bei gleichbleibendem Cortisolrhy thmus - nicht mehr vorhanden. Bei 2 weiteren Patienten ohne Prostatacarcinom, aber mit erhöhter Aktivität der Lactatdehydrogenase bzw. alkalischen Phosphatase, konnte ebenfalls eine Tageszeitabhängigkeit dieser Kenngrößen festgestellt werden.

\section{Diurnal variation of the elevated acid phosphatase activity in cases of prostate carcinoma}

Summary: The diurnal rhythm of total acid phosphatase and prostatic phosphatase activities was investigated in patients with prostate carcinoma. In these patients, the activities of total acid phosphatase, the tartrate-sensitive fraction of acid phosphatase, and lactate dehydrogenase decrease after therapy, whereas the activity of alkaline phosphatase increases. In all patients with prostate carcinoma, the total and tartrate-inhibited acid phosphatase, and the level of cortisol show a diumal rhy thm before therapy, with a minimum at night. In one patient, after orchiectomy, the cortisol rhy thm remained unchanged, but the daily phosphatase variation was absent. Diurnal variations of lactate dehydrogenase and alkaline phosphatase were also observed in 2 patients without prostate carcinoma, but with elevated levels of these enzymes.

\section{Einleitung}

Uber die circadiane Rhy thmik von Enzymaktivitäten im Serum liegen nur wenige Untersuchungen mit zum Teil widersprüchlichen Ergebnissen vor $(1,2,3)$. Bei Messungen um 8.00, 1.00 und $14.00 \mathrm{Uhr}$ konnte keine Tageszeitabhängigkeit bei gesunden Probanden für die Aspartataminotransferase, Alaninaminotransferase, alkalische Phosphatase und Lactatdehydrogenase ermittelt werden (4). Aus methodischen Gründen dürfte es zumindest bei Aktivitäten im Normbereich schwierig sein, geringfügige Zeitstrukturen zu ermitteln. Auf der anderen Seite konnte Deguchi (5) bei Versuchen an

\footnotetext{
1) Die Arbeit wurde unterstuitzt aus Mitteln der Robert Bosch Stiftung, Stuttgart.
}

Ratten zeigen, daß die Serotonin-N-Acetyltransferaseaktivität des Corpus pineale eine ausgeprägte circadiane Rhythmik mit einem Maximum gegen 19.00 Uhr zeigt. Allerdings konnte die Phasenlage durch verschiedene Einflüsse manipuliert werden.

In einer interessanten Untersuchung von $\mathrm{Doe} \& \mathrm{Mel}$ linger (6) wurde gezeigt, daß bei Prostatacarcinomen mit erhöhten Aktivitäten der gesamten sauren Phosphatase und der Prostataphosphatase eine ausgeprägte Rhythmik vorlag, die nach Therapie verschwand. Ziel unserer Studie war es, bei einigen Patienten diesen Befund zu überprüfen und zu untersuchen, ob andere Enzyme mit erhöhter Aktivität dasselbe Verhalten zeigen. Außerdem sollte durch Messung an Patienten ohne Prostatacarcinom mit erhöhten Aktivitäten der 
Lactatdehydrogenase oder alkalischen Phosphatase eine eventuelle Abhängigkeit der Meßwerte von der Tageszeit nachgewiesen werden. Als ,Marker" mit einer ausgeprägten Rhy thmik wurde das Cortisol mitgemessen.

\section{Material und Methoden}

Die Untersuchungen wurden bei 5 Klinikpatienten durchgeführt. 3 Patienten wurden wegen eines Prostatacarcinoms aufgenommen. Von diesen 3 Patienten konnten 2 vor und nach Therapie untersucht werden. Patient M. J. wurde zur Prostatekto mie in eine urologische Klinik verlegt, so daß eine Nachuntersuchung nicht möglich war. Bei dem Patienten T. J. wurde die zweite Untersuchung 4 Wochen nach der Orchiektomie durchgeführt. Patient N. P. wurde 14 Tage vor der zweiten Versuchsserie orchiektomiert und 10 Tage lang täglich mit $24 \mathrm{mg}$ Chlorotrianisen (1 Tablette Merbentul, Merrell) behandelt.

Der Patient T. K. war in stationärer Beobachtung wegen einer Erhöhung der Lactatdehydrogenase unbekannter Ursache bei Tumorverdacht. Der Patient E. A. war in stationärer Behandlung wegen einer seit mehreren Jahren bekannten alkoholischen Lebercirrhose. Von allen Patienten lag die Einwilligung für die durchgefuhrte Untersuchung vor. Die Blutentnahmen erfolgten über 24 Stunden im 3-Stunden-Abstand über einen liegenden Katheter aus der Kubitalvene, beginnend um 8.00 Uhr. Die Analysen der Serumproben erfolgten nach Beendigung der Versuchsserie. Bis zur Analyse wurden die Serumproben bei $+4{ }^{\circ} \mathrm{C}$ aufbewahrt und anschließend die Proben eines Patienten in Serie analysiert. Die Bestimmung der Aktivität der gesamten sauren Phosphatase und des Tartrat-hemmbaren Anteils der sauren Phosphatase erfolgte photometrisch durch Ermittlung der Spaltung von $p$-Nitrophenylphosphat bei $405 \mathrm{~nm}$ (Testkombination der Firma Boehringer Mannheim). Lactatdehydrogenase und alkalische Phosphatase wurden kinetisch mit einem Fast-Analyzer mit optimierten Methoden ermittelt (7). Cortisol wurde mit einer Proteinbindungsmethode bestimmt (8). Die Streuungen von Tag zu Tag der benützten Verfahren - ermittelt aus den Meßwerten der statistischen Qualitätskontrolle - liegen zwischen 5 und $10 \%$ bei einem Lagekriterium an der Entscheidungsgrenze. Die angegebenen Meßwerte der Patienten in den Abbildungen sind Mittelwerte aus Doppelbestimmungen.

\section{Ergebnisse und Diskussion}

In Tabelle 1 sind die Gleichwerte der verschiedenen Meßgrößen der einzelnen Probanden aufgeführt. Alle 3 Patienten mit Prostatacarcinom zeigen deutlich erhöhte Aktivitäten der gesamten sauren Phosphatase sowie des Tartrat-hemmbaren Anteils, der alkalischen Phosphatase und der Lactatdehydrogenase. Die beiden behandelten Patienten zeigen nach Therapie einen Abfall der Aktivitäten der gesamten sauren Phosphatase, des Tartrathemmbaren Anteils und der Lactatdehydrogenase.

Gleichzeitig kommt es zu dem bekannten Anstieg der alkalischen Phosphatase. In den Abbildungen 1, 2 und 3 sind die Ergebnisse der Messungen an den Patienten T. J. und N. P. vor und nach Therapie, sowie des 3 . Patienten M. J. mit Prostatacarcinom wiedergegeben.

Bei allen Patienten mit Prostatacarcinom zeigen gesamte saure Phosphatase und Tartrat-hemmbarer Anteil sowie Cortisol präoperativ bzw. vor Therapie eine Tageszeitabhängigkeit mit einem Minimum in der Nachtzeit. Dieser Befund stimmt mit den Ergebnissen von Doe \& $\mathrm{Mel}$ linger (6) überein. Diese Tageszeitabhängigkeit der genannten Meßgrößen ist bei dem Patienten T. J. (Abb. 1) nach der Orchiektomie - bei gleichbleibendem Cortisolrhythmus - nicht mehr vorhanden, wạhrend er bei dem Patienten N. P. (Abb. 2) für die drei genannten Größen bestehen bleibt. Allerdings ist auch der Therapieeffekt bei beiden Patienten unterschieḍlich; während es bei dem Patienten T. J. zu einem deutlichen Abfall der gesamten sauren Phosphatase und Normalisierung des Tartrathemmbaren Anteils kommt, ist deren Ak tivitätsabfall bei dem 2. Probanden (N. P.) erheblich weniger stark ausgeprägt. Der Verlauf der Aktivitäten von Lactatdehydrogenase und alkalischer Phosphatase ist sehr unterschiedlich. Während bei dem Patienten $\overline{\mathrm{T}}$. J.

Tab. 1. Gleichwerte der gemessenen Parameter der 5 untersuchten Patienten.

\begin{tabular}{|c|c|c|c|c|c|c|c|c|}
\hline Name & $\begin{array}{l}\text { Alter } \\
\text { (Jahre) }\end{array}$ & & $\begin{array}{l}\text { Saure } \\
\text { Phospha- } \\
\text { tase } \\
\text { gesamte } \\
\text { (U/1) }\end{array}$ & $\begin{array}{l}\text { Saure } \\
\text { Phospha- } \\
\text { tase } \\
\text { Tartrat- } \\
\text { hemmbare } \\
\text { (U/l) }\end{array}$ & $\begin{array}{l}\text { Alkalische } \\
\text { Phospha- } \\
\text { tase } \\
\text { (U/l) }\end{array}$ & $\begin{array}{l}\begin{array}{l}\text { Lactat- } \\
\text { dehydro- } \\
\text { genase }\end{array} \\
\text { (U/l) }\end{array}$ & $\begin{array}{l}\text { Cortisol } \\
(\mu \mathrm{g} / 1)\end{array}$ & Diagnose \\
\hline \multirow{2}{*}{ T. J. } & \multirow{2}{*}{68} & vor Therapie & 89 & 39 & 2150 & 419 & 73 & \multirow{2}{*}{$\begin{array}{l}\text { Prostata-Ca } \\
\text { Stadium IV }\end{array}$} \\
\hline & & nach Therapie & 38 & 4,4 & 6760 & 200 & 46 & \\
\hline \multirow{2}{*}{ N.P. } & \multirow{2}{*}{67} & vor Therapie & 118 & . 74 & 2450 & 572 & 318 & \multirow{2}{*}{$\begin{array}{l}\text { Prostata-Ca } \\
\text { Stadium II }\end{array}$} \\
\hline & & nach Therapie & 97 & 67 & 4070 & 175 & 151 & \\
\hline M. J. & $72^{\circ}$ & . & 134 & 112 & 257 & 184 & 109 & $\begin{array}{l}\text { Prostata-Ca } \\
\text { Stadium IV }\end{array}$ \\
\hline T. K. & 75 & & $14, \ddot{7}$ & 0,7 & 183 & 2640 & 201 & $\begin{array}{l}\text { Tumor- } \\
\text { verdacht }\end{array}$ \\
\hline E. A. & 75 & & 8,7 & 3 & 390 & 242 & 112 & $\begin{array}{l}\text { Leber- } \\
\text { cirrhose. }\end{array}$ \\
\hline
\end{tabular}




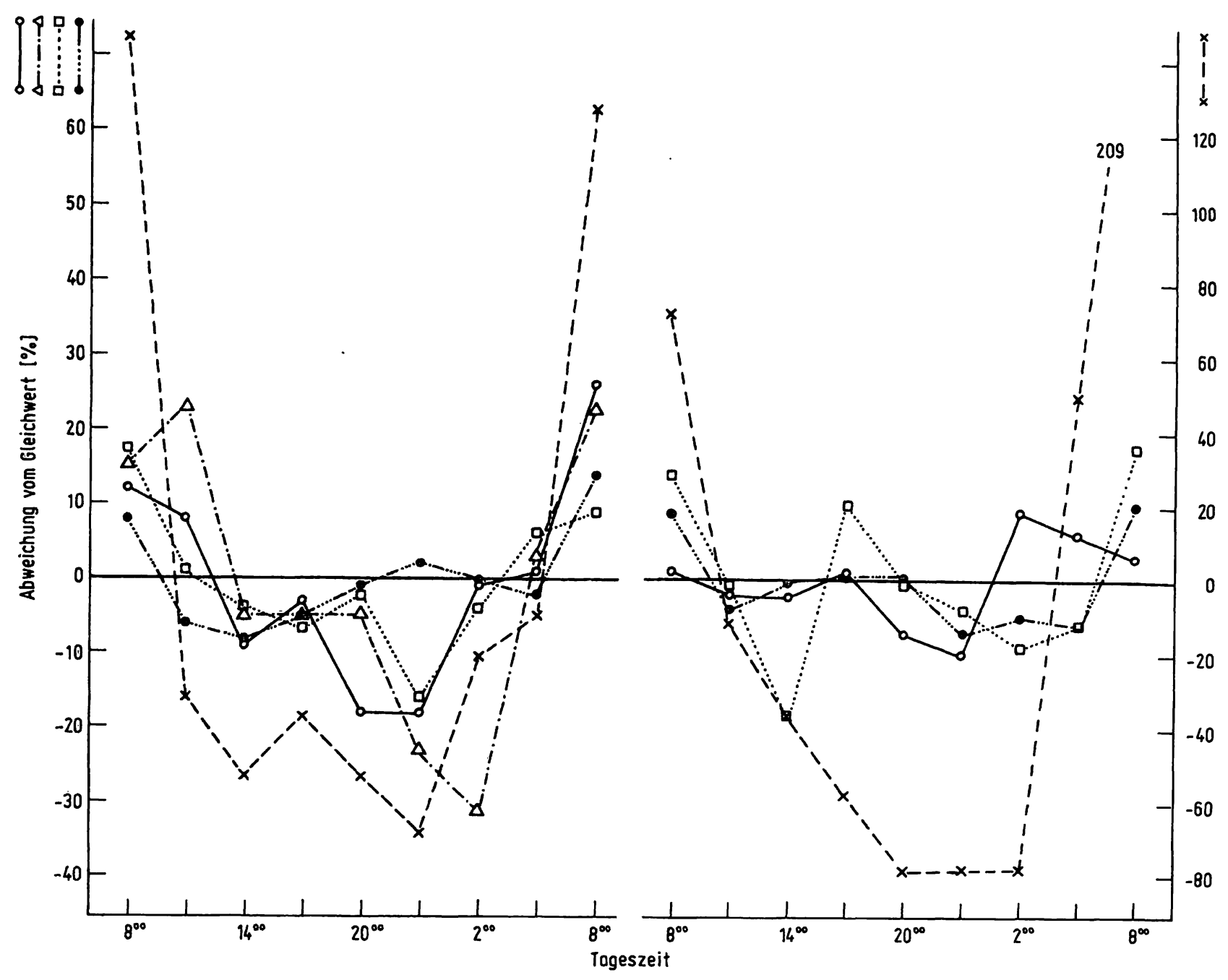

Abb. 1. Tageszeitabhängigkeit der gesamten alkalischen Phosphatase ( $0-0)$, der Tartrat-hemmbaren sauren Phosphatase ( $\Delta-\cdot-\cdot \Delta)$, der Lactatdehydrogenase $(0 \cdots \cdots . . \cdot$ ) und alkalischen Phosphatase $(\bullet \cdots-\cdots-\bullet)$ bei einem Patienten (T. J.) links vor und rechts nach Orchiektomie. Der Maßstab der Ordinate rechts gilt für das Cortisol $(x---x)$.
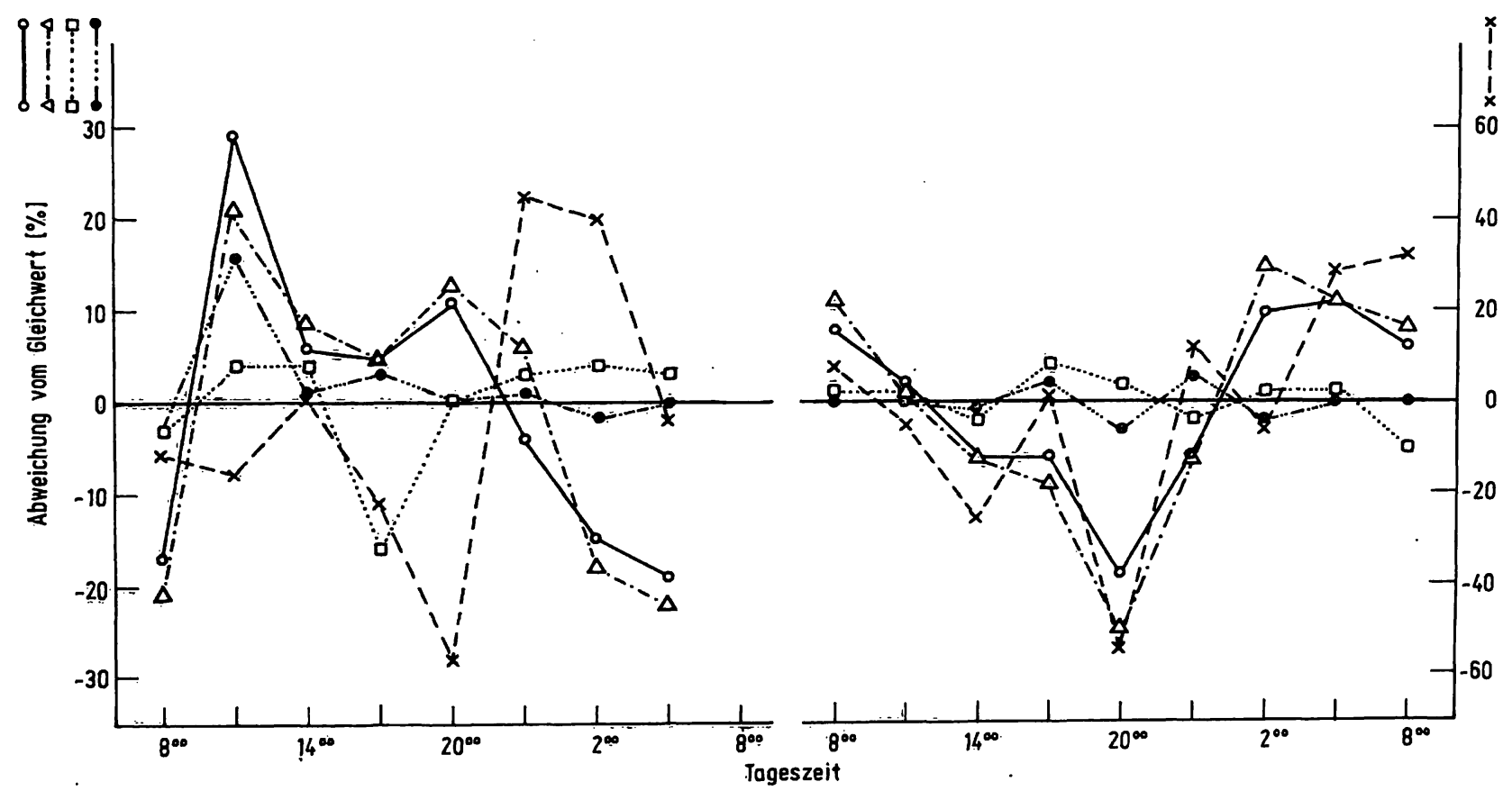

Abb. 2. Tageszeitabhängigkeit der gesamten alkalischen Phosphatase $(0-0)$, der Tartrat-hemmbaren sauren Phosphatase ( $\Delta-\cdot-\cdot \Delta)$, der Lactatdehy drogenase $(0 \cdots \cdots \cdots \cdot)$ und alkalischen Phosphatase $(0 \cdots-\cdots-\cdots)$ bei einem Patienten (N. P.) mit Prostatacarcinom links vor und rechts nach Therapie. Der Maßstab der Ordinate rechts gilt fựr das Cortisol $(x---x)$. 


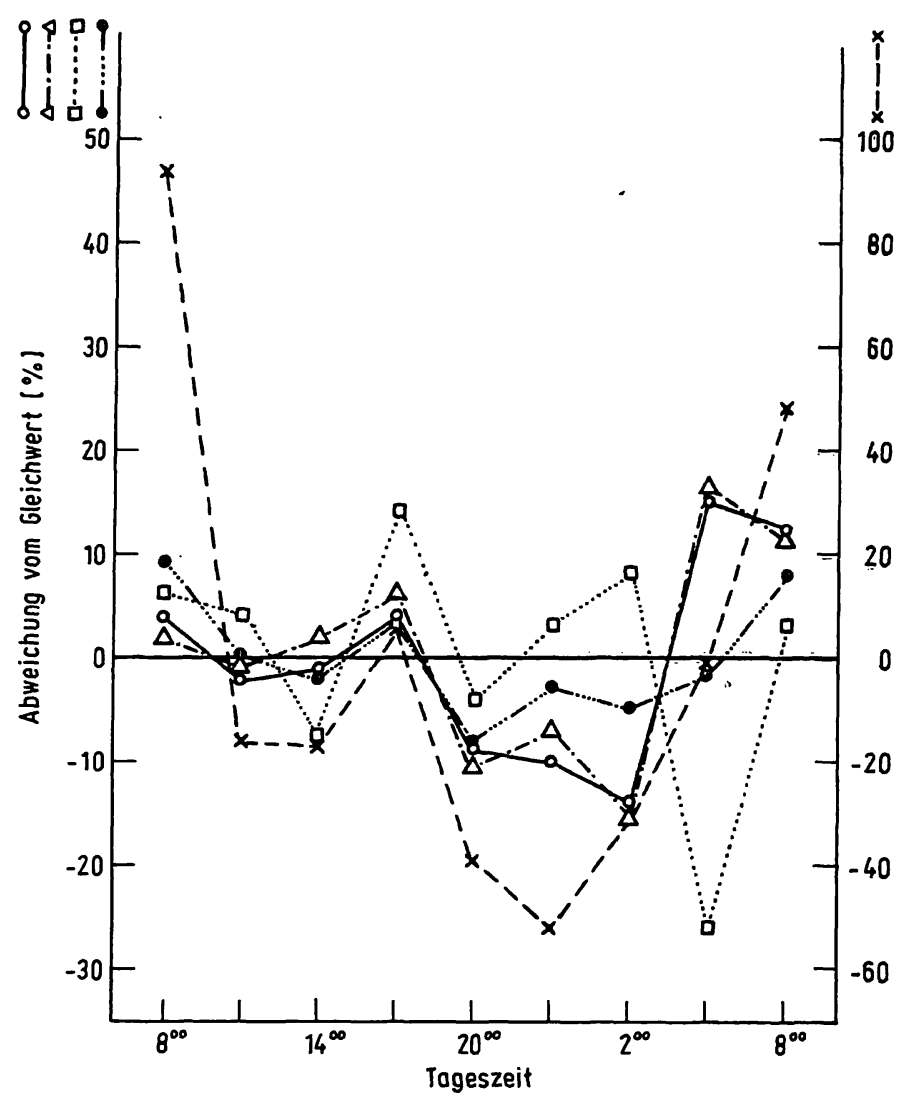

(Abb. 1) zumindest präoperativ die beiden Enzyme eine Ảbhängigkeit ihrer Aktivität von der Tageszeit zeigen, ist eine derartige Abhängigkeit bei den beiden anderen Patienten mit Prostatacarcinom nicht zu sehen.

Wie die Abbildung 4 zeigt, ist bei beiden Probanden mit einer stark erhöhten Aktivität von Lactatdehydrogenase 60 und einer Aktivität der alkalischen Phosphatase im Normbereich (Abb. 4 links) sowie bei normaler Lactatdehydrogenase bei mäßig erhöhter Aktivität der alkalischen Phosphatase (Abb. 4 rechts) eine Tageszeitabhängigkeit aller gemessenen Kenngrößen vorhanden. Die Ërgebnisse der Tartrat-hemmbaren sauren Phosphatase ergaben ein völlig irreguläres Verhalten. Allerdings dürfte im Normbereich der Tartrat-hemmbaren sauren Phosphatase der Meßfehler des benützten. Verfahrens zu groß sein, um wenig ausgeprägte Zeitstrukturen zu erkennen.

Abb. 3. Tageszeitabhängigkeit der gesamten alkalischen Phosphatase (०- $)$ ), der Tartrat-hemmbaren sauren Phosphatase $(\Delta-\cdot-\Delta)$, der Lactatdehydrogenase $(0 \cdots \cdots . . . \cdot)$ und alkalischen Phosphatase (๑.....--) bei einem Patienten (M. J.) mit Prostatacarcinom. Der Maßstab der Ordinate rechts gilt für das Cortisol $(x---x)$.
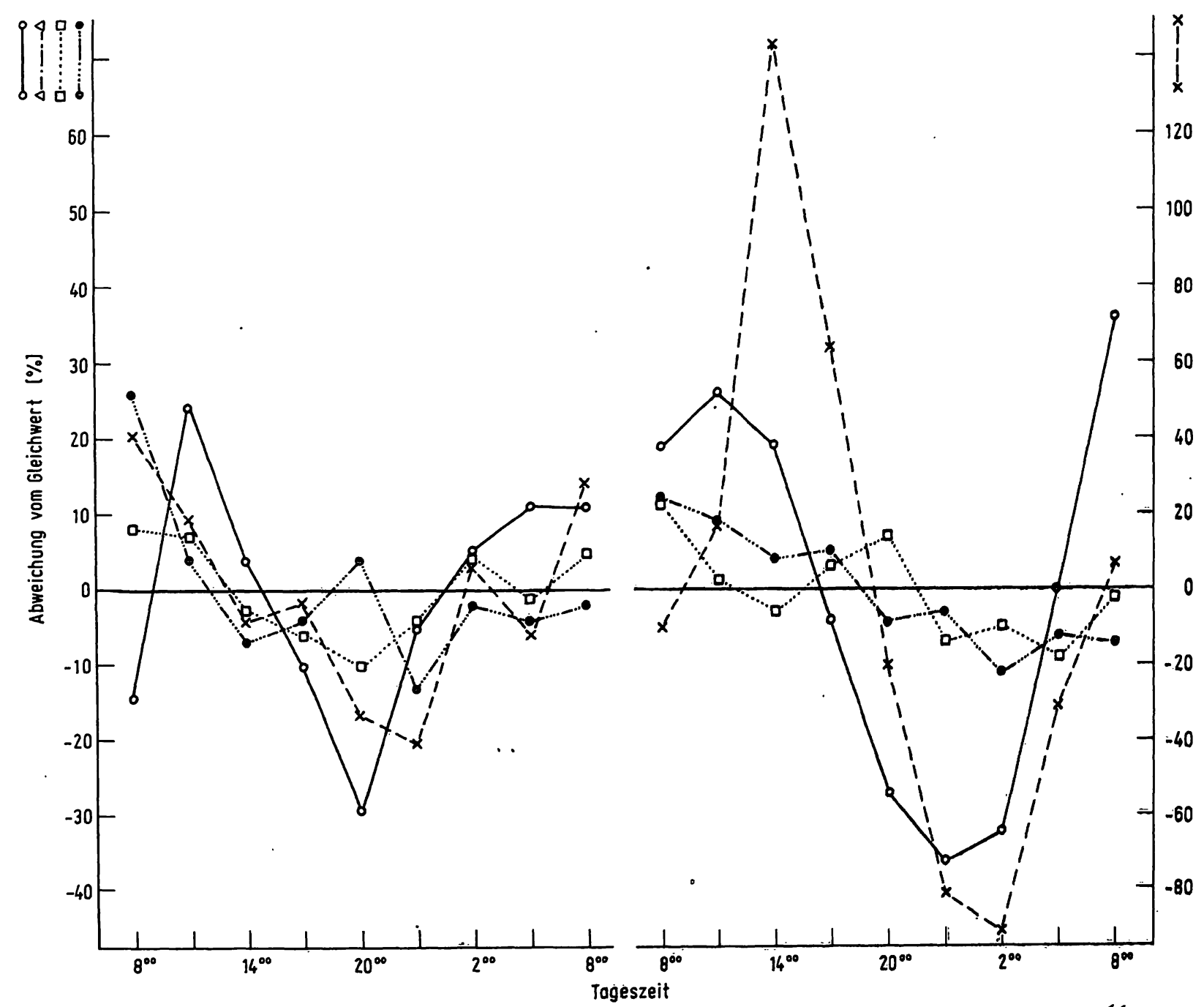

Abb. 4. Tageszeitabhängigkeit der gesamten alkalischen Phosphatase $(0-0)$, der Tartrat-hemmbaren sauren Phosphatase ( $\Delta-\cdot-\Delta)$,

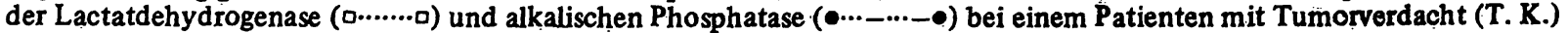
(links) und bei einem Patienten mit Lebercirrhose (E. A.) (rechts). Der Maßstab der Ordinate rechts gilt für das Cortisol (x-- - x). 
Auch ein radioimmunologisches Verfahren ${ }^{2}$ ) war für diese Fragestellung zu unempfindlich.

Ingesamt kann also festgestellt werden, daß die gemessenen Enzymaktivitäten eine Tageszeitabhängigkeit mit Schwingungsbreiten bis zu $40 \%$ und einem nächtlichen Minimum zeigen. Dies stimmt, was die Lactatdehydrogenase, gesamte und Tartrat-hemmbare saure Phosphatase angeht, mit den Ergebnissen anderer Untersucher überein $(3,6)$. Eine Aufhebung dieser Tageszeitabhängigkeit nach Therapie konnte bei den untersuchten Pro-

2) NEN Chemicals, Dreieich

\section{Literatur}

1. Massarat, S. (1964) Klin. Wochenschr. 42, 91-94.

2. Nuttall, F. Q. \& Jones, B. (1968) J. Lab. Clin. Med. 71, 847-854.

3. Jacey, M. J. \& Schaefer, K. E. (1968) Aerospace Medicine $39,410-412$.

4. Statland, B. E., Winkel, P. \& Bokelund, H. (1973) Clin. Chem. 19, 1374-1379. banden nicht eindeutig bestätigt werden. Bei allen 5 Probanden war der bekannte Cortisolrhythmus mehr oder minder stark ausgeprägt. Die Ergebnisse an 2 Probanden ohne Prostatacarcinom lassen ebenfalls eine Tageszeitabhängigkeit erkennen. Möglicherweise sind die z. T. widersprüchlichen Ergebnisse methodisch bedingt. Es könnte aber auch sein, daß es bei bestimmten Erkrankungen gegenüber gesunden Probanden zu einer Verstärkung der Tageszeitabhängigkeit durch Ausschaltung „störender" Faktoren kommt. Diese Möglichkeit soll durch Untersuchungen der Tageszeitabhängigkeit wichtiger Kenngrößen an Patienten mit verschiedensten Krankheitsbildern ermittelt werden.

5. Deguchi, T. (1977) Am. J. Physiol. 232, 375-381.

6. Doe, R. J. \& Mellinger, G. T. (1964) Metabolism 13, 445-452.

7. Empfehlungen der Deutschen Gesellschaft für Klinische

Chemie (1972) Z. Klin. Chem. Klin. Biochem. 10, 182-192.

8. Köbberling, J. \& v. z. Mühlen, A. (1972) Z. Klin. Chem. Klin. Biochem. 10,67-73.
Prof. Dr. Dr. H. Wisser Abteilung für Klinische Chemie Robert-Bosch-Krankenhaus Auerbachstr. 110 D-700 Stuttgart 50 


$$
\text { . }
$$

